\title{
Hybrid Cat Swarm and Crow Search Algorithm to Solve the Combined Economic Emission Dispatch Model for Smart Grid
}

\author{
Raj Kumar \\ Department of Mechanical Engineering \\ Cape Institute of Technology \\ Tirunelveli, Tamil Nadu, India \\ krajkumark@gmail.com
}

\begin{abstract}
In power system, economic load dispatch is a significant optimization issue. Because of the destructive effects of the emission released by fossil fueled electric power plants. In power plants, Emission Dispatch plays a vital role. It leads to the formulation of combined economic and emission dispatch (CEED) issue. In this work, a CEED model is introduced for the smart grid system and it resolves using hybridization of the well-known optimization approaches like Cat Swarm Optimization (CSO) and Crow Search Algorithm (CSA). The hybridization of these two well-known methods is attained exploiting the updating memory procedure of both the approaches. The CEED model is exploited to reduce the cost in order to sufficient trade-off among the emission and economic costs can be maintained in reducing them. Moreover, the developed algorithm is simulated on 3 different bus systems and its performance is analyzed over individual Particle Swarm Optimization (PSO), Grey Wolf Optimization (GWO) and Differential Evolution (DE) and a lately framed hybridization of CSO and CSA. The evaluation analysis exhibits the dominance of the proposed hybrid method regarding the CEED cost model.
\end{abstract}

Keywords: Economic Dispatch; Power System; CEED; Cost Model; Emission Model; Optimization

\begin{tabular}{ll} 
Nomenclature \\
\hline Abbreviations & Descriptions \\
\hline ED & Economic Dispatch \\
SDP & Semi-Definite Programming \\
EED & Economic Emission Dispatch \\
IPM & Interior Point Methods \\
DED & Dynamic Economic Dispatch \\
NBI & Normal Boundary Intersection \\
GA & Genetic Algorithm \\
CEED & Combined Emission ED \\
DE & Differential Evolution \\
WSM & Weighted Sum Method \\
PSO & Particle Swarm Optimization \\
BF & Bacterial Foraging \\
CDF & Cumulative Distribution Function \\
HS & Harmony Search \\
PDF & Probability Density Function \\
SR & Spinning Reserve \\
MODE & Multi-Objective DE \\
PEVs & Plug-in Electric Vehicles \\
GSA & Gravitational Search Algorithm \\
V2G & Vehicle-to-Grid \\
PDE & Pareto DE \\
NSGA-II & Non-Dominated Sorting GA-II \\
G2V & Grid-to-Vehicle \\
SPEA-2 & Strength Pareto Evolutionary Algorithm 2 \\
PHEVs & Plug-in Hybrid Electric Vehicles \\
ABC & Artificial Bee Colony \\
EVs & Electric Vehicles \\
\hline
\end{tabular}

(C)Resbee Publishers

https://doi.org/10.46253/jcmps.v2i3.a2 


\begin{tabular}{ll}
\hline EMOCA & Enhanced Multi-Objective Cultural Algorithm \\
CDC & Count Dimension to Change \\
BSA & Backtracking Search Algorithm \\
ACO & Ant Colony Optimization \\
SPC & Self Position Consideration \\
FA & Firefly Algorithm \\
SRD & Seeking Range Dimension \\
BA & Bat Algorithm \\
AP & Awareness Probability \\
SMP & Seeking Memory Pool \\
\hline
\end{tabular}

\section{Introduction}

In power systems, ED is a significant task, whose aspire is to discover the optimal scheduling to reduce the total fuel cost in certain constraints for generators. In addition, emission must be incorporated with the growing examined on the environment. This subject to an increase in the examination of EED that is fors0oth a multi-objective optimization issue. As the EED is merely appropriate to a definitely fixed time interval, the DEED is developed that aspires at reducing emission and operation cost concurrently in a 24-hour time spans [2].

The DED requires to examine ramping constraints among various time periods that create the issue increasingly strenuous to resolve. Nevertheless, due to its scheduling scheme is practical, the DED has received huge attention. Global warming and environmental pollution possess become more and more important; a great challenge in emission minimization and energy-saving appears in most countries in current years. To attain emission minimization and energy saving, the conventional economic scheduling with the aim to reduce the generation cost is slowly changed into a multi-objective EED that needs to reduce both pollution emission and generation cost concurrently [24]. Moreover, DEED is the hybridization of EED and DED [1]. An alternate method to attain emission minimization and energysaving is to link much increasingly renewable energy sources, like wind power, into the electric grid, therefore, the emission, and generation of existing thermal units, can be minimized.

Generally, the DEED solution technique has been categorized into an intelligent approach and conventional techniques. The conventional techniques like SDP [18] and IPM [17] techniques are exploited to resolve the multi-objective DEED issue, however to attain a collection of Pareto solutions, processing algorithms such as NBI or linear WSM are used to transform multiple objectives into a single objective, and their solution programs required to be termed multiple times with diverse weights. The conventional approaches possess the benefits of maximum effectual and rapid speed, and there are so far many commercial solvers present for researchers. Nevertheless, its solution is sensitive to the primary value, and if the objective model is non-convex or not differentiable, the approach might not work.

CEED is another solution that does not need any speculation. CEED only alters the output power of generators to require the load demand with minimum acceptable emission rate and production cost. By solving CEED needs an innovative algorithm to offer practical solutions. To solve CEED with operation constraints finding innovative algorithms is an important topic that has received huge attraction from academia and industry. In recent years [9-13] metaheuristic algorithms, as well as Artificial intelligence, were developed. In non-linear systems, these methods possess more appropriate performance while comparing with the conventional algorithms. Nevertheless, they mainly have inappropriate search exploration in the existence of a high number of local optima. The speed of convergence, as well as precision of evolutionary algorithms, are other significant properties which require to be contemplated in practice [19].

The optimization algorithms such as GA [8], DE [10], PSO [9], HS [11], Flower Pollination [12], MODE [13], GSA [18], PDE [13], NSGA-II [13], SPEA-2 [13], ABC-PSO [14], EMOCA [15], FA [16], BA [16], Cuckoo Search based algorithms [30-32], ACO [33], Gradient-based Jaya algorithm [17], BSA [20] and other nature based on the algorithms was stated for CEED optimization. In large-scale complex systems, many of aforesaid algorithms were slow computation time and lower speed of convergence. Practical systems have the superior curse of non-convex, dimensionality, and non-linear constraints, which instantly trouble the performance of evolutionary and classical algorithms. Additionally, optimum operation of a system with minimum emission and fuel costs is the important criterion for Energy Management System (EMS) and power grid enterprises for CEED.

This main aim of this work presents a CEED model for the smart grid. Additionally, a hybridized optimization model is presented to resolve the CEED model. Here, the CEED model is considered emission and economic constraints so that sufficient trade-off can be maintained among these two constraints. In addition, the proposed method comprises network and security constraints of the smart 
grid for secure and reliable operation for the power system. The developed optimization method is a hybridization of CSO and CSA algorithm.

\section{Literature Review}

In 2019, Gang Liu et al [1], worked on the evaluation of the uncertain nature of wind power output, the Weibull distribution parameters of regional wind speed at diverse periods were computed to attain the $\mathrm{CDF}$ and PDF the of the wind power. For wind power integration, the SR requirements were determined based on the attained CDF and PDF, and by converting the wind power into a possibility constrained form, a model for DEED with wind power was modeled. A hybrid multi-objective method that combines PSO and DE method was to forward an idea to resolve the developed method. The algorithm was developed on the basis of a dynamic external archive set and Pareto dominance theory, and completely uses the benefits of PSO and DE.

In 2018, Ming-Tse Kuo et al [2], developed an algorithm in order to determine the ED in power systems during the process of carbon emissions. To assure operational constraints and load demands, conventional ED was determined by the reduction of total power generation costs on the basis of the power output of the generating units in a system. In the developed model, carbon emissions were exploited. $\mathrm{A} \mathrm{CO}_{2}$ corresponding method was generated which considered for the several fuel categories, for example, oil, natural gas, and coal exploited in power generation. The correlation between power carbon emissions and generation costs was decided on the basis of the incremental cost and $\mathrm{CO}_{2}$ emissions tradeoff $-\mathrm{CO}_{2}$ minimization curves. The presented algorithm was used to the Taiwan Power Company system.

In 2018, Huijun Liang et al [3], developed a novel issue on examining the DEED like PEVs for valley filling and peak shaving. Moreover, the effect caused by exploiting diverse G2V and V2G loads on DEED was evaluated. The optimization model of the issue was modeled as well as numerous practical constraints, like ramp rate limits and power flow constraints. The battery degradation cost was also incorporated that reshapes the objective model of DEED. On the basis of the model, an approach for DEED and PEVs for valley filling and peak shaving was subjected, and a multi-objective optimization method was used to resolve the presented issue.

In 2019, E. Arriagada et al [4], developed a probabilistic ED model. The developed approach contemplates uncertainties, affecting the small-term control, the load dispatching and emission factors. Solar radiation, wind speed, and power demand were treated as random variables. Additionally, unavailability factors were considered. The solution scheme was on the basis of the Monte Carlo algorithm and a bi-objective linear optimization constrained process. The algorithm consists of multidimensional probabilities, bimodal analysis and clusters study descriptive statistics. The optimal solution produces the probability distributions of dual costs, system marginal prices, and emission factors, renewable and thermal power generation, and load-shedding.

In 2017, Mohammad-Reza Andervazh and Shahram Javadi [5], worked on the incorporation of renewable energy resources and PHEVs into the power system, the environmental-Ed of existing power generation units gets original dimensions because of the various ineluctable uncertain factors. This work proposes a probabilistic optimization model for the emission, ED of thermal power generation units, taken into consideration the stochastic charging demand of PHEVs, intermittent uncertain load demand and wind power generation. The Nataf transformation and point estimate algorithm were used to characterize the uncertainty in the output variables, by taking into consideration the correlation among wind power plants. To discover Pareto optimal solutions regarding the reduction of the fuel cost and emission, heuristic fitness sharing methods, and a meta-heuristic technique were presented.

In 2019, Md Samar Ahmad and S. Sivasubramani [6], worked on the transportation network, which was predictable to be subjugated using EVs. The power grid requires augmenting its generation ability to meet their charging demand with an augment in grid linked vehicles. Hence, it was necessary to discover the maximum number of $\mathrm{EVs}$ which was the conventional infrastructure be able to handle from a grid perception. This work presents a novel model to recognize the fleet size ability of a power network in both $\mathrm{V} 2 \mathrm{G}$ and uncontrolled modes of operation. The developed technique was designed as an optimization issue and examined a system that was created by integrating IEEE 33-bus Radial Distribution System (RDS) as well as a ten-unit generation system.

In 2017, Mostafa Kheshti [7], worked on the significance of environmental impacts and global warming which collected from the emission of gaseous pollutants of fossil-fuelled power plants, the modern CEED was exploited. This work presents a novel evolutionary lightning flash method to resolve dual objective CEED issues by taking into consideration of the different cases with multiple fuel options, wind power penetration, and operation constraints on the generators. The lightning flash method was presented on the basis of the movements of the cloud to ground lightning strikes in a thunderstorm. 
Finally, this algorithm was examined on eleven benchmark models and subsequently it was used in 6 different practical case study systems in order to solve the non-convex CEED.

\section{Combined Economic Emission Dispatch Model for Smart Grid}

\subsection{Cost Model}

The cost model is the conventional economic load dispatch model in that diverse generator kinds are presented to decide the economic manner in order to meet the power demand. Consider, $\mathrm{G}_{\mathrm{u}}$ generating units and the cost model $\mathrm{C}_{1}(\bullet)$ is stated in eq. (1).

$$
\mathrm{C}_{1}\left(\mathrm{G}_{1}\right)=\sum_{\mathrm{l}=1}^{\mathrm{N}_{\mathrm{l}}} \sum_{\mathrm{h}=0}^{\mathrm{N}_{\mathrm{a}}-1} \mathrm{c}_{\mathrm{h}+1}\left(\mathrm{G}_{1}\right)^{\mathrm{h}}
$$

In eq. (1), $N_{a}$ denotes the number of cost coefficients, c denotes a set of cost coefficients, and $\mathrm{G}_{1}$ indicates the generating limits of the $1^{\text {th }}$ generating unit. Therefore, $G_{1}$ is presented to meet the generation capacity constraint as stated in eq. (2). Here, $\mathrm{G}_{1}^{\max }$ and $\mathrm{G}_{1}^{\min }$ are maximum and minimum generation capacities, correspondingly. As the cost model is taken into consideration as the quadratic coefficient, $N_{a}$ it has become 3 .

$$
\mathrm{G}_{1}^{\min } \leq \mathrm{G}_{1} \leq \mathrm{G}_{1}^{\max }
$$

Additionally, the $\mathrm{G}_{1}$ must meet the real power balance constraint with the generation capacity constraints,

$$
\sum_{l=1}^{N_{1}} G_{1}-\left(D_{P}+T_{L}\right)=0
$$

In eq. (3), $\mathrm{T}_{\mathrm{L}}$ and $\mathrm{D}_{\mathrm{P}}$ indicate the transmission losses and power demand correspondingly. By exploiting eq. (4), the $\mathrm{T}_{\mathrm{L}}$ can be determined in that $\mathrm{A}, \mathrm{A}^{\prime}$ and $\mathrm{A}^{\prime \prime}$ are loss coefficients.

$$
\mathrm{T}_{\mathrm{L}}=\sum_{\mathrm{l}_{1}=1}^{\mathrm{N}_{\mathrm{l}}} \sum_{\mathrm{l}_{2}=1}^{\mathrm{N}_{\mathrm{l}}} \mathrm{G}_{\mathrm{l}_{1}} \mathrm{~A}_{\mathrm{l}_{1} \mathrm{l}_{2}} \mathrm{G}_{\mathrm{l}_{2}}+\sum_{\mathrm{l}=1}^{\mathrm{N}_{\mathrm{l}}} \mathrm{A}_{1}^{\prime} \mathrm{G}_{1}+\mathrm{A}^{\prime \prime}
$$

\subsection{Emission Model}

The emission model indicates the quantity of greenhouse gas emissions happen indefinite period, while the generating units are in operation. Therefore, eq. (5) is used to represents the emission model $\mathrm{C}_{2}(\bullet)$.

$$
\mathrm{C}_{2}\left(\mathrm{G}_{1}\right)=\sum_{\mathrm{l}=1}^{\mathrm{N}_{\mathrm{l}}} \sum_{\mathrm{h}=0}^{\mathrm{N}_{\mathrm{E}}-1} \beta_{\mathrm{h}+1}\left(\mathrm{G}_{1}\right)^{\mathrm{h}}
$$

In eq. (5), $\mathrm{E}_{\mathrm{C}}$ represents the number of emission coefficients and $\beta$ represents a set of emission coefficients. As same to the cost model, the emission model is a quadratic equation and therefore $\mathrm{E}_{\mathrm{C}}$ has become 3 .

\subsection{CEED Model}

The general CEED model tries to reduce the summation of the emission and cost models which are subjected to meet the constraints presented in eq. (2) to (4). Therefore, it can be resolved as a constrained reduction issue. This work transforms it as an unconstrained minimization issue $\mathrm{F}_{\mathrm{u}}(\bullet)$ in that the constraints are involved in the minimization model with appropriate penalty factors as stated in eq. (6).

$$
\mathrm{F}_{\mathrm{u}}\left(\mathrm{G}_{1}\right)=\mathrm{F}\left(\mathrm{G}_{1}\right)+\delta_{1}\left|\mathrm{P}_{\mathrm{b}}\right|+\delta_{2}\left|\mathrm{P}_{\mathrm{L}}\right|
$$

In eq. (6), $\mathrm{P}_{\mathrm{b}}$ indicates the real power balance constraint exploited in the LHS of the eq. (3). In the primary two terms of the CEED model indicates the emission model and the cost model, correspondingly, in contrast, the third and fourth phrase indicates the network and security constraints, correspondingly. On the basis of the CEED model, the objective model can be formulated as eq. (7).

$$
\mathrm{G}_{1}^{*}=\underset{\mathrm{G}_{1}}{\arg \min } \mathrm{F}_{\mathrm{u}}\left(\mathrm{G}_{1}\right)
$$


In eq. (7), $\mathrm{G}_{1}^{*}$ indicates the optimal power to be generated using the generating units under minimized cost, emission and transmission losses.

\section{Proposed Crow Search and Cat Swarm methodology for CEED model}

The hybrid CSO and CSA algorithm are used to solve the CEED model; CSO and CSA are clearly stated in this section.

\subsection{CSO}

The conventional CSO method is a swarm intelligence approach that is enthused by the cat's behavior [21]. Here, two modes are considered on the behavior of cats, tracing mode and seeking mode. In the seeking mode, $\mathrm{N}$ cats move from their locations gradually and close to their origin positions in $\mathrm{D}$ dimensional space, $\mathrm{y}_{1, \mathrm{~d}}$ whereas $\mathrm{l}=\{1,2, \ldots \ldots, \mathrm{N}\}$ and $\mathrm{d}=\{1,2, \ldots \ldots, \mathrm{D}\}$. Additionally, a cat moves on the basis of its own velocity in tracing mode. In each mode, a ratio of the mixture $\mathrm{m}_{\mathrm{r}}$ is exploited to decide the ratio number of cats distributed. A flag is inclined to assist in labeling if a cat is in the tracing or seeking mode.

\section{a) Seeking Mode:}

A global search is applied in the search space in the seeking mode. There are 4 parameters that are SPC where $\mathrm{SPC} \in\{0,1\} \mathrm{SRD}, \mathrm{SMP}$, and CDC whereas CDC and $\mathrm{SRD} \in\{0,1\}$.

In the seeking mode for each $l^{\text {th }}$ cat flagged, the steps are explained as below:

i) For each cat, the examination is done for the SPC parameter value. If the SPC of $\mathrm{m}^{\text {th }}$ cat $=1$ subsequently produces (SMP -1) copies of $\mathrm{m}^{\text {th }}$ the cat and maintains the source location. Moreover, produces SMP copies.

ii) To alter the positions use a mutation operation. According to the CDC, decide the number of dimensions to be altered. Alter the $\mathrm{i}^{\text {th }}$ selected dimension at a time step $\mathrm{t}$ by exploiting eq. (8).

$$
\mathrm{y}_{1, \mathrm{i}}^{\mathrm{t}+1}=\mathrm{y}_{1, \mathrm{i}}^{\mathrm{t}}+(-1)^{\mathrm{i}} * \mathrm{SRD} * \mathrm{y}_{1, \mathrm{i}}^{\mathrm{t}+1}
$$

iii) Estimate the fitness $f_{i}$

iv) In archive save the location

v) Use Roulette Wheel selection [22] for each copies cat $l=\{1, \ldots . ., \mathrm{SMP}\}$ by exploiting eq. (9).

$$
\begin{aligned}
\mathrm{P}_{1} & =\frac{\left|\mathrm{f}_{1}-\mathrm{f}_{\mathrm{b}}\right|}{\mathrm{f}_{\max }-\mathrm{f}_{\text {min }}} \\
\mathrm{R}_{1} & =\frac{\mathrm{p}_{1}}{\sum_{1} \mathrm{p}_{1}} \\
\mathrm{C}_{1} & =\mathrm{C}_{1-1}+\mathrm{R}_{1}
\end{aligned}
$$

In eq. (9), $P_{1}$ is the selected probability of $1^{\text {th }}$ copy, $f_{b}: b$ is high if the objective is reduction and or else $b$ is low, $C_{1}$ represents the cumulative probability of $1^{\text {th }}$ copy and $R_{1}$ represents the relative probability of $1^{\text {th }}$ copy. Produce arbitrarily $r_{1} \in[0,1]$ if $r_{1} \leq \mathrm{C}_{1}$ subsequently the initial cat is selected. In addition, select copy $\mathrm{l}^{\text {th }}$ if $\mathrm{C}_{1-1}<\mathrm{r}_{1} \leq \mathrm{C}_{1}$.

\section{b) Tracing Mode:}

In the CSO approach, local search optimization is indicated using the tracing mode. The acceleration coefficient $\left(\mathrm{a}_{\mathrm{c}}\right)$ is exploited for expanding the velocity. A cat possesses a maximum need for tracing targets. The process of tracing mode is stated as below:

i) Using eq. (12), update the velocities:

$$
\mathrm{u}_{1, \mathrm{~d}}^{\mathrm{t}+1}=\mathrm{u}_{1, \mathrm{~d}}^{\mathrm{t}}+\mathrm{a}_{\mathrm{c}} * \mathrm{r} *\left(\mathrm{y}_{\text {best,d }}-\mathrm{y}_{1, \mathrm{~d}}^{\mathrm{t}}\right)
$$

ii) Using eq. (13), update the location of $1^{\text {th }}$ cat.

$$
\mathrm{y}_{1, \mathrm{~d}}^{\mathrm{t}+1}=\mathrm{y}_{1, \mathrm{~d}}^{\mathrm{t}}+\mathrm{u}_{1, \mathrm{~d}}^{\mathrm{t}+1}
$$

iii) Accesses the fitness $\left(f_{1}\right)$

iv) In archive save the location 


\subsection{CS Algorithm}

CS is an evolutionary approach on the basis of the flocks of crow's behavior [23]. Generally, crows are called as highly intelligent birds. They observe and examine whereas other birds conceal their food. Subsequently, the crow takes the food while the owner leaves. Crows have better self-awareness; they will move their concealing places to evade a rustler. Crows have better memorized, even in a couple of months afterward they will recall their concealing place up. The modifiable parameters of the CS method are flight length $\left(f_{1}\right)$ and AP. The process of the CS algorithm for $N$ number of crows in $D$ dimensional problem is stated as follows:

i) Initialize location $\left(y_{1, d}\right)$ and memory of crows $\left(c_{1, d}\right)$, whereas $l=\{1,2, \ldots . . N\}$ and $d=\{1,2, \ldots . . D\}$. Due to the crows still have no knowledge's in this primary mode; it is supposed the source location as their initial memories.

ii) Estimate fitness.

iii) Arbitrarily select one of the crows to go after, for instance, $\mathrm{m}^{\text {th }}$ crow. Update the new locations of the $1^{\text {th }}$ crow by exploiting eq. (14).

$$
\mathrm{y}_{1, \mathrm{~d}}^{\mathrm{t}+1}= \begin{cases}\mathrm{y}_{1, \mathrm{~d}}^{\mathrm{t}}+\mathrm{r}_{1} * \mathrm{f}_{\mathrm{l}} *\left(\mathrm{c}_{\mathrm{m}, \mathrm{d}}-\mathrm{y}_{1, \mathrm{~d}}^{\mathrm{t}}\right) & \mathrm{r}_{1} \geq \mathrm{AP}_{\mathrm{m}} \\ \mathrm{a} \quad \text { random position } & \text { O.W }\end{cases}
$$

In eq. (14), $\mathrm{r}_{1}$ produce randomly with uniform distribution among zero and one. The AP of crow $\mathrm{m}$ is indicated using the $\mathrm{AP}_{\mathrm{m}}$. This situation denotes that crow $\mathrm{m}$ understands that crow $\mathrm{l}$ is following, to guard its food, the crow $\mathrm{m}$ will move arbitrarily in search spaces to track it.

iv)Calculate the fitness of new locations.

v) Update the memory of $1^{\text {th }}$ crow while the fitness value $\left(f_{i}\right)$ of the memorized position is not superior adequate comparing with the new location.

\subsection{Proposed Hybrid CSO-CS Algorithm}

In Fig. 1, the flowchart of the hybrid CSO-CS algorithm is demonstrated. For each $1^{\text {th }}$ individual $(l=1,2, \ldots, N)$, the process of the hybrid method is stated as follows:

i) Adjustable parameters and initialize problem. Parameters are a number of individuals $\left({ }^{N}\right)$, SMP, CDC, SRD, mixing ratio $\left(\mathrm{m}_{\mathrm{r}}\right)$, acceleration coefficient $\left(\mathrm{a}_{\mathrm{c}}\right)$, awareness probability $(\mathrm{AP})$, and flight length $\left(f_{1}\right)$.

ii) Arbitrarily initialize the velocity, location, and memory of an individual. $\mathrm{N}$ individuals arbitrarily located in $\mathrm{D}$ dimensional space whereas $\mathrm{y}_{\mathrm{l}, \mathrm{d}}$ indicating the location of the individual $\mathrm{l}$ in the dimension $\mathrm{d}, \mathrm{u}_{1, \mathrm{~d}}$ indicating velocity and $\mathrm{c}_{1, \mathrm{~d}}$ indicating memory. At first, it is supposed that the initial location as the initial memory [23].

iii) For each individual, estimate the fitness (objective) function.

iv) Give labeling self-location consideration $\mathrm{SPC}=1$ to an individual $l$ if $\mathrm{y}_{\mathrm{l}, \mathrm{d}}$ it is the optimal solution $\left(\mathrm{y}_{\text {best }}\right)$. In addition to providing $\mathrm{SPC}=0$, or else.

v) Based on the $\mathrm{m}_{\mathrm{r}}$, arbitrarily picked individuals from the population as well as their flag gets on with it seeking mode. Remnants the flag gets on with tracing mode.

vi) For individuals using the seeking mode procedure that are in seeking mode by the description from the preceding part.

vii) Gather the new individual location in the archives from the tracing mode and seeking mode.

viii) Update the memory while the fitness model value of the new location is superior to the fitness model value of the memorized location.

ix) Using eq. (14), produce a new location from the selected copy in seeking mode and location in the tracing mode.

$\mathrm{x})$ For each individual, estimate the fitness (objective) function.

xi) Update the memory while the fitness model value of the memorized location is not superior adequate when compared with the new location.

xii) Ensure the termination condition. While the termination is fulfilled, the optimal location in memory is stated as the optimal solution. else, repeat steps (iv) -(xii). 


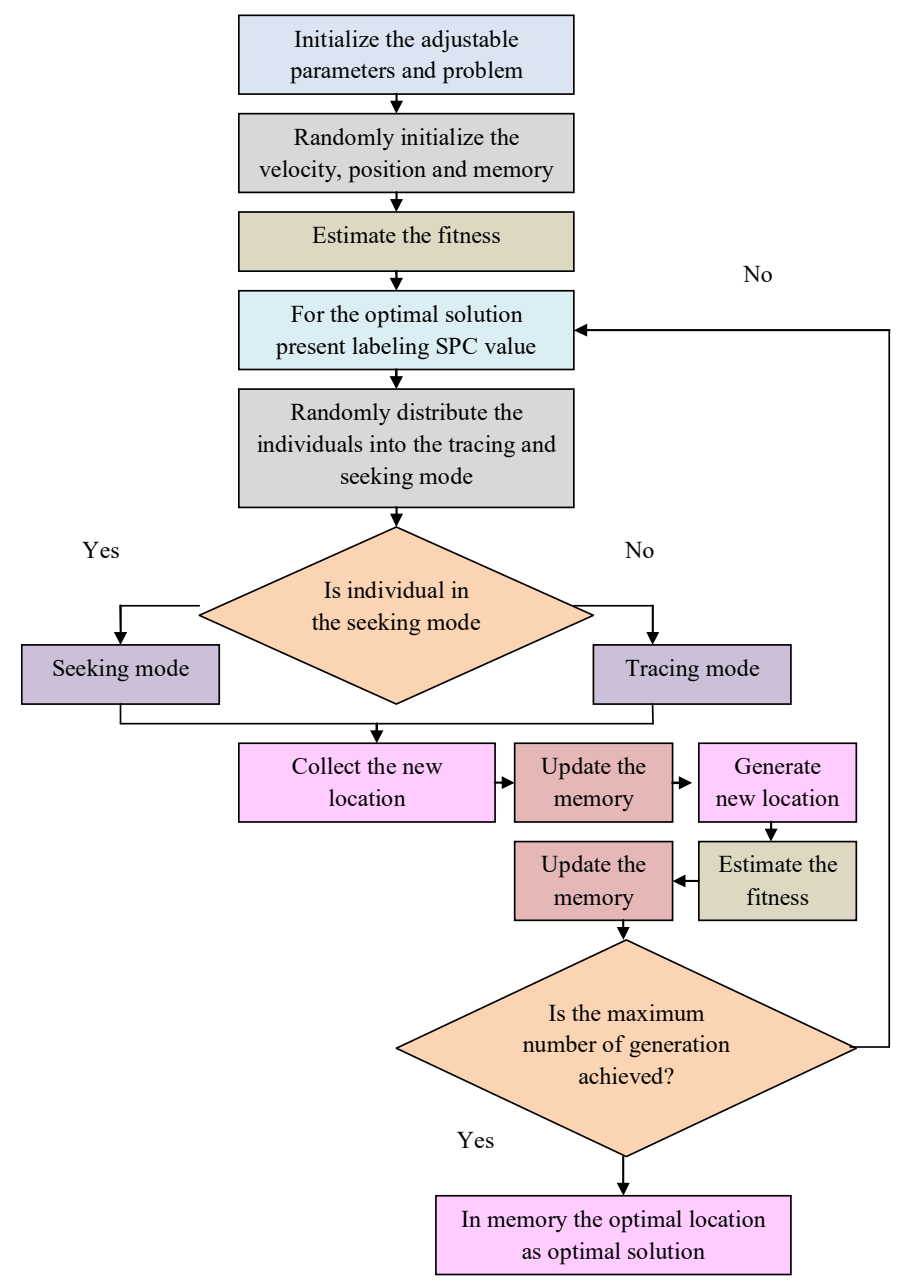

Fig.1: Flow chart of the proposed hybrid CSO-CS algorithm

\section{Results and Discussions}

\subsection{Simulation Setup}

The developed CEED model and the hybrid model have been performed in MATLAB and the performance analysis was done on three test systems. Here, the first and second test systems have three and six different types of generators, and the third is the IEEE 30 bus system correspondingly. Moreover, the proposed technique was evaluated with traditional DE, PSO and GWO techniques.

\subsection{Performance Analysis}

The analysis of the optimization algorithms on minimizing the CEED cost can be seen in Fig. 2,3 and 4 . Here, the methods performed better than the conventional PSO, DE and GWO algorithms it is on the basis of the achieved costs for their generation scheme. For the three generator bus systems, the proposed model has attained the best cost as it has generated the minimum cost by its generation schedule, while the existing techniques, such as DE, GWO, and PSO have attained high cost than the proposed method and it is shown in Fig 2. Similar type of performance is attained under the examination of the median and worst cost achievement and it is shown in Fig 3 and 4. Nevertheless, the proposed algorithm could attain only less in accomplishing the worst cost throughout the iterations. 


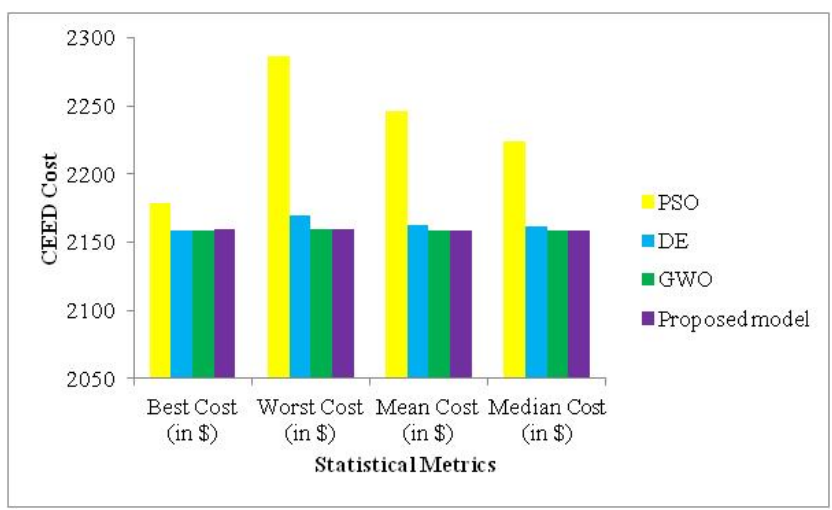

Fig.2: Performance analysis of the proposed and conventional algorithms regarding the minimized CEED cost utilized in the 3 generator bus system

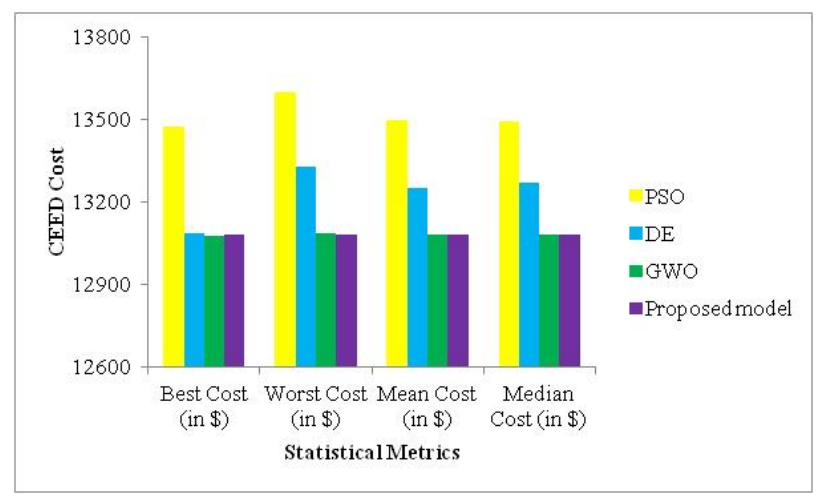

Fig. 3: Performance analysis of the proposed and conventional algorithms regarding the minimized CEED cost utilized in the 6 generator bus system

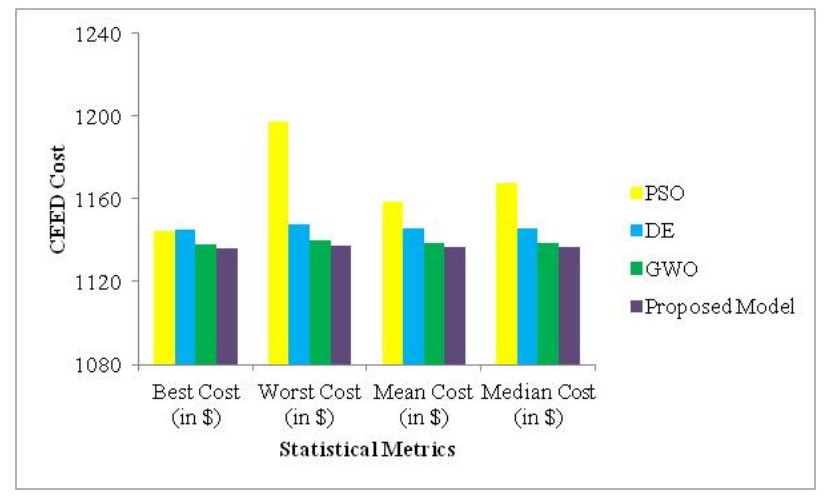

Fig. 4: Performance analysis of the proposed and conventional algorithms regarding the minimized CEED cost utilized in the IEEE 30 bus system

\section{Conclusion}

For the smart grid system, to solve the CEED model, this paper presents the hybridization of the optimization algorithms such as CSO and CSA. Here, the hybridization was performed using integrating the memory update process of CSA, and CSO. The CEED model has considered emission and economic constraints besides with transmission and security constraints. The simulation was performed on a 3 generator bus system and six generator bus systems. Here, the 6 generator bus systems were the benchmark in IEEE 30 bus systems. The outcomes were exposed that the proposed technique was superior to the existing techniques. At last, the proposed technique regarding the superior performance of the proposed hybrid technique was assured by the analysis of the minimized cost of CEED. 


\section{Compliance with Ethical Standards}

Conflicts of interest: Authors declared that they have no conflict of interest.

Human participants: The conducted research follows the ethical standards and the authors ensured that they have not conducted any studies with human participants or animals.

\section{References}

[1] G. Liu, Y. L. Zhu and W. Jiang, "Wind-thermal dynamic economic emission dispatch with a hybrid multiobjective algorithm based on wind speed statistical analysis," IET Generation, Transmission \& Distribution, vol. 12, no. 17, pp. 3972-3984, 3092018.

[2] M. Kuo, S. Lu and M. Tsou, "Considering Carbon Emissions in Economic Dispatch Planning for Isolated Power Systems: A Case Study of the Taiwan Power System," IEEE Transactions on Industry Applications, vol. 54, no. 2, pp. 987-997, March-April 2018.

[3] H. Liang, Y. Liu, F. Li and Y. Shen, "Dynamic Economic/Emission Dispatch Including PEVs for Peak Shaving and Valley Filling," IEEE Transactions on Industrial Electronics, vol. 66, no. 4, pp. 2880-2890, April 2019.

[4] E. Arriagada, E. Lopez, M. Lopez, G. Lefranc, R. Lopez and M. Poloujadoff, "A Probabilistic Economic/CO2eq Emissions Dispatch Model: Real Applications," IEEE Latin America Transactions, vol. 16, no. 9, pp. 2362-2369, September 2018.

[5] M. Andervazh and S. Javadi, "Emission-economic dispatch of thermal power generation units in the presence of hybrid electric vehicles and correlated wind power plants," in IET Generation, Transmission \& Distribution, vol. 11, no. 9, pp. 2232-2243, 2262017.

[6] M. S. Ahmad and S. Sivasubramani, "Optimal Number of Electric Vehicles for Existing Networks Considering Economic and Emission Dispatch," in IEEE Transactions on Industrial Informatics, vol. 15, no. 4, pp. 1926-1935, April 2019.

[7] M. Kheshti, X. Kang, J. Li, P. Regulski and V. Terzija, "Lightning flash algorithm for solving non-convex combined emission economic dispatch with generator constraints," in IET Generation, Transmission \& Distribution, vol. 12, no. 1, pp. 104-116, 212018.

[8] Basu, M.: 'Economic environmental dispatch of fixed head hydrothermal power systems using nondominated sorting genetic algorithm-II', Appl. Soft Comput., 2011, 11, pp. 3046-3055

[9] Wang, L., Singh, C.: 'Reserve constrained multi-area environmental/ economic dispatch based on particle swarm optimization with local search',Eng. Appl. Artif. Intell., 2009, 22, pp. 298-307.

[10] Bhattacharya, A., Chattopadhyay, P.K.: 'Solving economic emission load dispatch problems using hybrid differential evolutions', Appl. Soft Comput., 2011, 11, (2), pp. 2526-2537.

[11] Chatterjee, A., Ghoshal, S.P., Mukherjee, V.: 'Solution of combined economic and emission dispatch problems of power systems by an opposition-basedharmony search algorithm', Int. J. Electr. Power Energy Syst., 2012, 39, pp. $9-20$

[12] Abdelaziz, A.Y., Ali, E.S., Abd Elazim, S.M.: 'Combined economic and emission dispatch solution using flower pollination algorithm', Int. J. Electr.Power Energy Syst., 2016, 80, pp. 264-274.

[13] Basu, M.: 'Economic environmental dispatch using multi-objective differential evolution', Appl. Soft Comput., 2011, 11, pp. 2845-2853.

[14] Manteaw, E.D., Odero, N.A.: 'Combined economic and emission dispatch solution using ABC_PSO hybrid algorithm with valve point loading effect', Int. J. Sci. Res. Publ., 2012, 2, (12), pp. 1-9

[15] Zhang, R., Zhou, J., Mo, L., et al.: 'Economic environmental dispatch using an enhanced multi-objective cultural algorithm', Electr. Power Syst. Res., 2013, 99, pp. 18-29

[16] Gherbi, Y.A., Bouzeboudja, H., Gherbi, F.Z.: 'The combined economic environmental dispatch using new hybrid metaheuristic', Energy, 2016, 115,pp. 468-477

[17] Vo, D.N., Schegner, P., Ongsakul, W.: 'Cuckoo search algorithm for nonconvex economic dispatch', IET Gener. Transm. Distrib., 2013, 7, pp. 645-654.

[18] Zhou, J., Wang, C., Li, Y., et al.: 'A multi-objective multi-population ant colony optimization for economic emission dispatch considering power system security', Appl. Math. Model., 2017, 45, pp. 684-704

[19] Azizipanah-Abarghooee, R., Dehghanian, P., Terzija, V.: 'Practical multi-area bi-objective environmental economic dispatch equipped with a hybrid gradient search method and improved Jaya algorithm', IET Gener. Transm. Distrib., 2016, 10, (14), pp. 3580-3596

[20] Modiri-Delshad, M., Abd Rahim, N.: 'Multi-objective backtracking search algorithm for economic emission dispatch problem', Appl. Soft Comput., 2016, 40, pp. 479-494.

[21] S. P. Chu, S. C. Chu, P. W. Tsai, and J. S. Pan, "Cat Swarm Optimization”, LNAI 4099, 3 (1), Berlin Heidelberg: Springer-Verlag, 2006, pp. 854-858.

[22] S. N. Deepa dan S. N Sivandanam, "Introduction to Genetic Algorithms", Springer, India, 2007, pp. 47-48.

[23] A. Askarzadeh, "A Novel Metaheuristic Method for Solving Constrained Engineering Optimization Problems: Crow Search Algorithm", Computers and Structures 169, 2016, pp. 1-12.

[24] Chithra.S and R.Madahana Kumari," Emission Dispatch in Renewable Energy Systems using FireFly Algorithm"Journal of Computational Mechanics, Power System and Control (JCMPS), Volume 1, Issue 1, October 2018. 